\title{
Enfermería y paciente terminal: aspectos bioéticos
}

\author{
Bioethics and Nursing Care in Terminal \\ Patient \\ Dr. Francisco Javier León Correa* \\ *Doctor en Filosofía, Magíster en Bioética. Profesor Adjunto, Centro de Bioética, Pontificia Universidad Católica de Chile.
}

\section{Resumen}

La atención de Enfermería a los pacientes en situación terminal es importante, tanto por su intervención técnica clínica-sobre todo en la implementación de los cuidados paliativos-, como por la compleja tarea de acompañar al enfermo en afrontar su propia muerte. Es un deber ético de la enfermera prepararle junto con el médico y la familia, en proporcionarle la mejor calidad de vida posible, y defender también en esa situación los derechos del paciente, con un rol activo en el consenti- miento informado, así como en la toma de decisiones y juicios de proporcionalidad de los tratamientos. Se analiza en este trabajo la bibliografía existente tanto en el ámbito anglosajón como latinoamericano acerca del papel de Enfermería en la atención al paciente Terminal.

Palabras Clave: bioética, enfermería, enfermo Terminal

\section{Abstract}

Infirmary care of terminal patients is important, both because of it's clinical, technical intervention -above all in implementing paIliative care- and the complex task of accompanying the patient in facing his or her own death. It is an ethical must of the nurse to help the patient along with the physician and the patient's family, provide the patient with the best possible quality of life, and also defend the rights of the patient under those circumstances, with an active role in the informed consent, as well as in making decisions and judgments regarding treatments.

This work analyzes the existing bibliography both in the Anglo-Saxon and Latin American fields about the role of Infirmary in the care of terminal patients.

Key words: bioethics, nursing care, terminal patient

\section{INTRODUCCIÓN}

Trataremos en ste artículo de delimitar cuáles son los problemas y las posibles soluciones a los dilemas éticos que se le presentan a Enfermería en la atención a pacientes terminales. Aunque parte importante de las decisiones que han de tomar los equipos de salud en este campo corresponderán al médico o al equipo médico, no es menos cierto que las consecuencias de esas decisiones afectan de manera muy clara al quehacer diario y a la conciencia de las enfermeras que deben cuidar y atender a esos pacientes'.

Por otro lado, "la enfermera se encuentra identificada con los más altos valores morales y sociales de la humanidad y comprometida con aquellos que en particular propician una convivencia de dignidad, justicia e igualdad"2
Es propio de enfermería "el proceso de cuidar que se centra en las interrelaciones humano-humano, con una serie de conocimientos muy bien diferenciados, valores, dedicación, relación moral e interpersonal con el sujeto de cuidado"3. Es decir, con una ética del cuidado que debe tener en cuenta siempre el mejor bien del paciente 4 . El Consejo Internacional de Enfermería señala: "Al dispensar los cuidados la enfermera promoverá un entorno en el que se respeten los derechos humanos, valores, costumbres y creencias espirituales de la persona, la familia y la comunidad"5 y al referirse al final de la vida, comenta: "la función de la enfermera es fundamental para unos cuidados paliativos destinados a reducir el sufrimiento y a mejorar la calidad de la vida de los pacientes moribundos y de sus familias mediante una 
pronta evaluación, identificación y gestión del dolor y de las necesidades físicas, sociales, psicológicas, espirituales y culturales"6

Además, en la fase final de la vida "disminuye lentamente la función del médico y se hace más importante la de la enfermera: pasa a ser una importante fuente de información y consulta, y toma cada vez más la iniciativa" 7 .

Son situaciones que producen un gran impacto emocional tanto en el paciente, como en la familia y en el propio equipo sanitario que ha estado intentando su curación y debe cambiar desde ese momento de enfoque y finalidad de su actuación. Y en esto juega un papel muy importante e0nfermería, no sólo en la implementación de los cuidados paliativos -ya muy desarrollados por enfermeras en algunos países-, sino en la toma de decisiones ético-clínicas en esos momentos, como se ha destacado en la bibliografía norteamericana ${ }^{8,11}$ y como comienza a destacarse en el ámbito europeo y latinoamericano, con estudios desde la percepción de las propias enfermeras ${ }^{12,16}$. No se trata solamente de resguardarse de modo pasivo ante posibles responsabilidades legales ${ }^{17}$, sino de participar activamente en el proceso de decisiones para ayudar a afrontar de modo humano y digno esa situación al paciente y su familia18, 19 .

\section{DESARROLLO: DEBERES ÉTICOS PARA CON EL PACIENTE TERMINAL \\ Preparar al paciente para enfrentar la muerte con dignidad: respeto a la autonomía}

Es éste uno de los cometidos de todo el equipo sanitario, pero especialmente de la enfermera, que deberá realizarlo de acuerdo con la familia y con las sucesivas etapas emocionales de adaptación, que se presentan en mayor o menor medida en todos los casos: deseos de conocer o de confirmación del diagnóstico, shock con fuerte conmoción interna, fase de negación, enfado, negociación o pacto, depresión, resignación y aceptación final 20,21

El médico debe informar el diagnóstico, pronóstico y tratamiento al paciente, de modo general, pero es misión de la enfermera comprobar que el paciente lo ha entendido, y ayudarle a asimilar la información. En los actuales Códigos de ética de Enfermería está muy presente la importancia de la actuación directa de la enfermera para resolver los problemas éticos que se presentan en la atención, en su papel de promover la autonomía del paciente: "a través del consentimiento informado se protege y hace efectiva la autonomía de la persona que es absolutamente esencial en los cuidados de enfermería"2,11. "La enfermera debe verificar que las personas (paciente, familia, acompañante) reciban información suficiente para aceptar o rechazar los cuidados y tratamientos"22.

También puede superar la barrera de distancia que con frecuencia se interpone entre los médicos y los pacientes, y establecer un diálogo con tiempo suficiente para desarrollar una relación de ayuda, que normalmente el médico no puede realizar.

Los pacientes terminales experimentan una serie de inquietudes con preguntas que en ocasiones no se atreven a hacer ni a sí mismos; temores que expresarán en la medida en que encuentren enfermeras a su lado que sean sensibles y comprendan lo que subyace en sus comentarios y dudas; dependencias físicas y psíquicas que pueden desmoralizarles y hacerles perder su propia autoestima y dignidad personal. En definitiva buscan encontrar un sentido a lo que les ocurre, precisamente a ellos. Pueden y deben encontrar en las enfermeras que les atienden una gran ayuda para adaptarse a ese proceso del morir, a esa despedida de sus seres queridos, antes de entrar en la inconsciencia. $Y$ las enfermeras deben estar preparadas, profesionalmente también, para prestar este cuidado plenamente humano, en condiciones más difíciles de atención al paciente ${ }^{23}$.

En definitiva, se trata de saber disponer al paciente para una muerte afrontada con dignidad. Para esto se necesita resolver una serie de puntos:

1. Lo primero es que el paciente sepa que se está muriendo. De este modo podrá resolver sus asuntos, tomar sus disposiciones, cumplir sus promesas o simplemente despedirse. Se tiende con excesiva frecuencia a ocultar el diagnóstico por parte de la familia, con la aquiescencia del médico, y es deber de la enfermera en esos casos intervenir a favor del propio paciente con prudencia, pero con claridad. Puede haber excepciones, pero no deben convertirse en la regla genera ${ }^{24}$

2. Es necesario que el enfermo pueda seguir siendo, en cierto modo, agente, y no mero paciente. Por ello debe intervenir en las decisiones sobre su enfermedad o sobre su persona. Es tarea de la enfermera comprobar que efectivamente ha comprendido la información dada por el médico, antes de que el paciente firme el protocolo de consentimiento, si no lo ha hecho en la consulta, como suele ser frecuente. En gran parte depende de las enfermeras que el consentimiento sea un proceso de información y no un mero trámite burocrático en nuestros hospitales ${ }^{25}$ 
3. Hay que preservar en la medida de lo posible la autonomía y autocontrol del paciente. La sensación de menor dignidad resulta de la excesiva dependencia y la pérdida de control de las funciones orgánicas.

4. Deberá mantener en la medida de lo posible ciertas actividades y relaciones familiares, sociales y profesionales. Sentirse persona implica ser capaz de relacionarse..

5. Se debe proteger la pérdida de la imagen corporal. Hay que evitar que el enfermo se sienta desfigurado o depauperado. Muchas veces esos cuidados los realizará la propia familia o auxiliares, pero la enfermera debe supervisarlo.

6. Al enfermo se le debe facilitar el apoyo espiritual si lo desea, y la enfermera puede facilitar al paciente o a sus familiares la información.

En este sentido, solamente apuntaremos que no debe confundirse el "morir con dignidad" o el "derecho a una muerte digna" con la eutanasia, con decidir el momento de la propia muerte. Es un debate diferente.

\section{ÉTICA DEL TRATAMIENTO PALIATIVO DEL DOLOR}

Cuando ya no es posible curar al paciente, queda en primer plano el aliviar su sufrimiento, que es éticamente obligatorio y justifica plenamente las actuaciones paliativas ${ }^{26}$, que son válidas si consiguen tal finalidad ${ }^{27}$.

La enfermera que trata enfermos en situación terminal tiene obligación de estar bien entrenada en el manejo de los diversos niveles de analgesia y aplicar en cada caso el conveniente para la situación del enfermo ${ }^{28}$. Si no consigue un eficaz control del dolor o se requieran técnicas especializadas debe poner al enfermo en manos del especialista médico indicado.

Cuando el enfermo se encuentra moribundo y el objetivo es conseguir el máximo control del dolor, se pueden utilizar pautas de sedación en las que exista la capacidad de despertar y llevar o seguir una conversación lúcida. Existe la obligación ética de no privar de la conciencia de sí mismo sin verdadera necesidad. Sin embargo, cuando se pretende la supresión de la sensación dolorosa, si existen motivos serios, se puede evitar el dolor, aunque lleve consigo una supresión o disminución de la conciencia. Si de la aplicación de algún fármaco se siguiera un acortamiento de la vida como efecto no buscado, no habría tampoco inconveniente en administrarlo ${ }^{29}$.
Algunos autores anotan que los opiáceos, por su efecto depresor del centro respiratorio, podrían facilitar la aparición de infecciones respiratorias. Por el contrario, otros señalan que globalmente alargan el tiempo de vida: "evidencias circunstanciales, sugieren que el uso correcto de la morfina prolonga la vida del paciente con cáncer en la medida en que se ve libre del dolor, en mejor situación para descansar, dormir y comer y, generalmente, se siente y está más activo" 30 .

En resumen, lo que se pretende es dar la atención adecuada al enfermo, que le lleve a tener la mejor calidad de vida posible en un ámbito hospitalario o, mejor aún, en su propio domicilio, evitando la angustia y soledad de las $\mathrm{UCI}^{31}$

\section{EL PACIENTE TERMINAL EN CUIDADOS INTENSIVOS Cuidados mínimos y medidas de soporte vital}

El enfermo ingresado en una $\mathrm{UCl}$, sigue precisando de cuidados médicos y atención de enfermería. Ser paciente en la UCI significa soledad, falta de comunicación. Junto a esto, sensación de seguridad y ser atendido por profesionales competentes, pero es una situación de dolor, angustia, sed y soledad, que debe tratar de humanizar la enfermera 32.

Los cuidados otorgados a un paciente en estado terminal no son aplicados como medios para preservar la vida, sino que se aplican en razón de una asistencia éticamente obligada, que debe buscar sobre todo la humanización de esa fase final de la vida del paciente, aún cuando pueda permanecer y fallecer en la $\mathrm{UCl}^{33}$.

Los médicos han de tomar las medidas necesarias para curar y conseguir la mejor calidad de vida con el enfermo recuperable, y también las decisiones de limitación de tratamientos, o pase del paciente a unidades de cuidados paliativos. Pero las enfermeras también cumplen aquí un papel importante. El juicio de proporcionalidad que realiza el médico no puede ser completo -porque no todo son los datos clínicos- sin el paralelo juicio de proporcionalidad que debe realizar al mismo tiempo el paciente o la familia, según sus valores, creencias, situación familiar, económica, etc.

La enfermera es el profesional más preparado para hacer de intermediaria del paciente, conocer su situación familiar y sus deseos, y hacer llegar la información precisa al médico, antes de la toma de decisiones ${ }^{34}$ También debe velar por el cumplimento de las voluntades anticipadas del paciente, si existiesen ${ }^{35}$.

Los cuidados de enfermería, cuando excepcionalmente han de ser aplicados por técnicas especiales, pueden perder su carácter de obligatoriedad y convertirse en cuidados 
extraordinarios o desproporcionados. Se estima que los cuidados básicos o mínimos para mantener la vida humana son la hidratación y la alimentación.

La hidratación por sonda nasogástrica o por vía endovenosa es habitualmente beneficiosa para un enfermo terminal. Es indudable que contribuyen al bienestar del paciente y ayudan psicológicamente evitando que se sienta abandonado. Reflejan cierto grado de preocupación por parte del personal sanitario, que el enfermo percibe. Sin embargo en el caso del paciente en estado agónico -que habitualmente es incapaz- se producen alteraciones fisiológicas que hacen difícil el equilibrio electrolítico, por lo que la hidratación por cualquiera de las vías no aportará beneficios y se convertirá en un cuidado extraordinario, tal vez solamente justificable para que la familia no considere que existe un abandono del moribundo. Ahora se aboga claramente por su suspensión cuando se está en un proceso agónico ya irreversible ${ }^{36}$

En el caso del paciente en estado vegetativo permanente (EVP) por lesión cerebral, la situación es muy distinta, pues no se trata de un paciente terminal en el sentido anteriormente definido, aunque sea incapaz. En este caso la hidratación por sonda o por vía endovenosa otorgará los beneficios antes señalados y las cargas para el paciente y el personal sanitario serán mínimas, por lo que podemos considerarlo siempre un tratamiento o un cuidado básico éticamente obligatorio ${ }^{37}$.

\section{La alimentación en el enfermo terminal}

Respecto a la alimentación se puede considerar como un cuidado mínimo, pero no necesariamente de cualquier modo. Sería un cuidado ordinario alimentar por sonda nasogástrica y si se espera una supervivencia razonable podría también incluirse la alimentación por gastrostomía. Sin embargo la alimentación parenteral habitualmente se puede considerar como un cuidado extraordinario, pues plantea mayores problemas de aplicación, su costo es elevado, el paciente ha de estar hospitalizado, debe ser controlada por la enfermera o el médico y no está exenta de riesgo.

El juicio de proporcionalidad de estos cuidados, recae tanto sobre la familia como sobre la enfermera, aunque la orden última es del médico.

Las enfermeras que atienden al paciente -y que deben contribuir con la tarea que desempeñan los nutricionistashan de tener en cuenta algunos principios ${ }^{38}$.

- Que conforme se acerca un paciente a la muerte se vuelve cada vez más desinteresado por la comida y, en menor medida, en la toma de líquidos.
- Que los inapetentes son incapaces de disfrutar de una comida abundante.

- Que sus necesidades calóricos son mucho menores.

- Que no está comprobado que una terapia nutricional agresiva en estos enfermos mejore su calidad de vida.

- Es necesario tener en lo posible en cuenta las preferencias del paciente, presentación y preparación de las comidas, horarios, dietas especiales, etc. Y también una forma importante de ayudarles es dar poco énfasis a su pérdida de peso. Pesar a los enfermos que están cada vez más delgados y anoréxicos resulta poco razonable.

\section{EL EMPLEO DE MEDIOS TERAPÉUTICOS "DESPROPORCIONADOS"}

¿Hasta qué punto hay que agotar con un enfermo todos los medios terapéuticos? ¿Es ético emplear curas costosas y difíciles aunque den solo una pequeña esperanza de éxito? ¿Es obligatorio poner todos los medios disponibles en cada caso, para mantener a un enfermo en vida el mayor tiempo posible? ¿Se puede o se debe prolongar artificialmente la vida?

No siempre hay que recurrir a toda clase de remedios posibles. Hasta ahora se hablaba de remedios "ordinarios" o "extraordinarios" para mantener la vida. Ahora, por la imprecisión del término y los rápidos progresos de la terapia, se prefiere hablar de medios "proporcionados" y "desproporcionados". Para realizar el juicio de proporcionalidad habrá que tener en cuenta el tipo de terapia, el grado de dificultad y de riesgo que comporta, los gastos necesarios, y las posibilidades de aplicación con el resultado que se puede esperar de todo ello, teniendo en cuenta las condiciones del enfermo y de sus fuerzas físicas y morales ${ }^{36,39,40}$ Con estos elementos habrá datos suficientes para decidir un tratamiento que prolongue la vida o para conformarse con los medios paliativos que la Medicina puede ofrecer., sin caer nunca en el encarnizamiento terapéutico.

En nuestra opinión el problema afecta más a enfermos que reciben terapias intensivas y sus familiares ${ }^{41,42}$, que a pacientes en situación Terminal en general, o que están en atención domiciliaria. Entre los profesionales de enfermería que se dedican a los cuidados paliativos, parece que estos dilemas están en parte resueltos en las guías clínicas ${ }^{43}$.

Aplicando el principio terapéutico o de beneficencia, que lleva a enjuiciar cada situación buscando el bien del enfermo, y el principio de autonomía, que lleva a respetar el derecho del paciente a intervenir en la toma de decisiones sobre el tratamiento a realizar, se puede encontrar una solución ética para cada situación concreta. 
Las decisiones sobre proporcionalidad tiene que tomarlas el médico, pero ya comentamos que la tarea de la enfermera es proporcionar los datos necesarios de la valoración subjetiva del paciente o la familia sobre las decisiones, así como los aspectos clínicos que desde enfermería piense deben tenerse en cuenta. La enfermera debe estar presente en esta toma de decisiones que no le competen exclusivamente al equipo médico. Está claro que cuando el paciente está en situación irreversible, no hay que agotar todos los medios terapéuticos, que no es ético el encarnizamiento terapéutico, que no es finalidad de la medicina mantener a un paciente con vida el mayor tiempo posible, sino por el contrario ayudar a que tenga la mayor calidad de vida posible en esos momentos finales de su vida.

Y aquí entran los cuidados paliativos como función primordial de la enfermera, junto a los demás profesionales, psicólogos, nutricionistas, kinesiólogos, fisioterapeutas.

La enfermera es, en los servicios de salud, la que lleva directamente la gestión de los cuidados. En Chile esto se ha reafirmado recientemente de modo expreso como misión de Enfermería ${ }^{44}$. Por tanto, debe unificar la gestión de los cuidados paliativos y participar previamente en las decisiones sobre limitación de tratamientos, para conseguir que efectivamente se comiencen los cuidados paliativos cuando son necesarios, sin que se produzca abandono del paciente, velando porque se respeten sus derechos.

\section{LOS DERECHOS DEL ENFERMO TERMINAL}

Los derechos del paciente son concreción de los derechos humanos primordiales, que fundamentan también la reflexión sobre valores y principios en Bioética. Tienen derecho ${ }^{45}$ : a recibir un cuidado especializado con el fin de minimizar los riesgos de morir o de quedar incapacitados; a obtener de sus médicos información acerca de su condición; a negarse a ciertos tratamientos dentro del marco permitido por la ley; a ser visitados por sus seres queridos, sin que esto interfiera en las regulaciones de cada $\mathrm{UCl}$; a recibir la asistencia religiosa por parte del ministro o sacerdote de su credo; a que se les ayude en gestiones de orden legal o personal que consideren importantes en su situación vital; a que toda información relativa al cuidado de su enfermedad sea tratada como confidencial; y finalmente, derecho a ser atendidos en forma personalizada, con especial referencia al trato respetuoso y considerado. Las enfermeras deben velar por el cumplimiento total cabal de estos derechos por parte de todos los profesionales que se encuentren realcionados con el paciente. ${ }^{46 .}$

\section{CONCLUSIONES}

La atención de Enfermería a los pacientes en situación Terminal es importante por su intervención clínica -sobre todo en la implementación de los cuidados paliativos-, y por la compleja tarea de acompañar al enfermo a afrontar su propia muerte. Es un deber ético de la enfermera prepararle a él y su familia, proporcionarle la mejor calidad de vida, y defender también en esa situación los derechos del paciente, con un rol activo en el consentimiento informado, en hacer valer las voluntades anticipadas que pueda haber tomado, así como en la toma de decisiones y juicios de proporcionalidad de los tratamientos.

Y es necesaria también una formación de la enfermera que le ayude a afrontar estos aspectos del sentido del dolor y la muerte, a establecer esa "relación de ayuda" imprescindible para la humanización de los cuidados en esos momentos tan importantes.

\section{REFERENCIAS BIBLIOGRÁFICAS}

1 Gómez-A; No me da miedo la muerte, me da miedo el dolor. Revista Index de Enfermería, Granada, 2007, 16 (58):6367.

2 Comisión Interinstitucional de Enfermería. Código de Ética para las enfermeras y enfermeros de México. México DF, 2001.

3 Código de Ética de la Enfermería colombiana. En: http://www. anec.org.co/

4 Torralba Francesc. Ética del cuidar. Fundamentos, contextos y problemas. Madrid: Institut Borja de Bioètica y Fundación Mapfre Medicina; 2002.5 Código de Ética del CIE para la profesión de Enfermería. Consejo Internacional de Enfermeras. Ginebra; 2006.

6 La función de la enfermera que dispensa cuidados a los pacientes moribundos y a sus familias. Declaración de posición del Consejo internacional de Enfermería, Ginebra, 2000, revisada 2006.

7 Van -Aren; J. Arie. La participación de la enfermera en la parte final de la vida. Enfermería Actualidad, Madrid, 1997 (9):12.

8 Childress Susan. Improving End-of-Life Care. Nursing Management, 2001, 32 10): 32-35.

9 Russell Sally. Nursing Care at the End of Life. American Academy of Ambulatory Care Nursing, Nov-Dec. 2003.

10 Fetter -S; Marilyn. End-of-Life Care: The Nursing Role. Revista Med Surg Bursing, 2000, 9: 230.

11 Ferrell Betty et al. End of Life Care: Nurses speak out. Nursing Ethics, 2000, 30 (7): 54-57.

12 López -P; Cinthia. Apoyo tanatológico a pacientes terminales. En: Identidad profesional de la enfermera ante la muerte. Revista IPN-CIECAS, México, 2004, no 7. http://www. ciecas.ipn.mx/ 
13 Hernández Mª E. La asistencia tanatológica. En: Identidad profesional de la enfermera ante la muerte. Revista IPNCIECAS, México, 2004, (7). http://www.ciecas.ipn.mx/

14 Hornos Josefina et al. Cuidados de enfermería al enfermo Terminal. Proyecto de una guía de acompañamiento a la muerte. Agathos: Atención sociosanitaría y bienestar. La Habana, 2006, 6 (4): 18-25.

15 Guevara B, Zambrano A, Evies A, Mejías M. Hacia un paradigma humanista en enfermería en la visión del enfermo terminal. Enfermería Global, revista electrónica. Murcia, España, 2005, 7. www.um.es/ojs/index.php/eglobal/5/ 05e01.html

16 Castillo Miguel A, Jiménez Ana I, Torres Imilla. Atención integral de enfermería al paciente oncológico en estadio terminal. Revista Cubana de Enfermería, 2007, 23 (2) http://bvs. sld.cu/revistas/enf/indice.html

17 Fernando Abellán, Sánchez-Caro. Enfermería y paciente: Cuestiones prácticas de bioética y derecho sanitario. Granada: Comares; 2007.

18 Feito Grande L. Ética Profesional de la Enfermería. Filosofía de la enfermería como ética del cuidado. Madrid: PPC; 2000.

19 Couceiro Azucena (ed.) Ética en cuidados paliativos. Madrid: Triascastela; 2004.

20 Cornago A. El paciente terminal y sus vivencias. Santander: Sal Terrae; 2007.

21 Kübler-R; Elisabeth, Kessler David. Sobre el duelo y el dolor. Barcelona: Editorial Luciérnaga; 2006

22 Código de Ética del Colegio de Enfermeras de Chile. Santiago, 2008. Capítulo 1, art. $3^{\circ}$.

23 Lautrette A, et al. A Communication Startegy and Brochure for Relatives of Patients Dying in the ICU. N Engl J Med 2007; 356: 469-78.

24 León Francisco J, Burattini Carla, Schwartzmann Jenniffer, Muñoz Mara. El consentimiento informado y el profesional de enfermería. En: Bioética e interculturalidad. Actas del I Curso Internacional y IV Jornadas de Bioética. Universidad de La Frontera, Temuco, Chile, 2008: 56-62.

25 León Francisco, Arratia Alejandrina. El consentimiento informado en hospitales chilenos. En: Bioética e interculturalidad. Ob.cit.: 62-67.

26 Espejo Dolores. Los cuidados paliativos. En: Pastor Luis M, León Francisco. Manual de ética y legislación en Enfermería. Madrid:Mosby-Doyma; 1997: 129-132.

27 Gracia Diego, Rodríguez -S; Juan J. (Dión). Ética en cuidados paliativos. Guías de ética en la práctica médica. Madrid: Fundación Ciencias de la Salud; 2006.

28 Avellaneda Luz S. Intervención de enfermería en los cuidados paliativos. Actual. Enferm. 2003; 6(3):22-28.

29 Clavé Eduardo. La sedación en cuidados paliativos. En: Astudillo Wilson, A., Casado A, Mendinueta C. Alivio de las situaciones difíciles y del sufrimiento en la terminalidad. San Sebastián, Sociedad Vasca de Cuidados Paliativos, 2005: 267-304.
30 Twycross A, Moriarty A et al. The management of chronic pain. En: Twycross A, ed. Paediatric pain management. Abingdon: Radcliffe Medical Press Ltd.;1998: 145-166.

31 Couceiro A. Tecnología, dolor y sufrimiento en las unidades de críticos. En: Dolor y sufrimiento en la práctica clínica. Barcelona: Humanitas, Humanidades Médicas, Monografía n 2, 2004: 53-67.

32 Llubià Maristany C. El poder terapéutico de la escucha en medicina crítica. Humanitas. Humanidades Médicas, Barcelona, 2008, 27. web: www.humanitas.es

33 Granja C et al. Patient's recollections of experiences in the intensive care unit may affect their quality of life. Crit Care 2005; 9: 145-6.

34 León Francisco, Espinoza Paz; López Marcela, Muñoz Mara. Orden de No Reanimación en Unidades de Cuidados Intensivos. Bioética e interculturalidad.Ob.cit.:

35 León Francisco, Abrigo Carolina, Délano Claudia, Muñoz Mara. Voluntades anticipadas en los pacientes terminales. El rol de Enfermería. Bioética e interculturalidad. Ob.cit.:

36 Gherardi C. Vida y muerte en terapia intensiva. Biblos, Buenos Aires, 2007: 113-128.

37 Shand B. Nutrición en los pacientes en estado vegetativo: un desafío pendiente. En León Francisco (coord.) Comisiones Nacionales de Bioética. Sociedad Chilena de Bioética, Santiago, 2008:107-109.

38 Sánchez -L; Miguel Anel et al. Intervención de Enfermería: herramienta clave para mejorar el estado nutricional en los pacientes. Revista SEDEN. 2007, 10 (4). www.revistaseden.org

39 Gómez -R; Juan A. Ética en Medicina crítica. Triacastela, Madrid, 2002

40 De la Torre Javier (ed.) La limitación del esfuerzo terapéutico. Comillas, Madrid 2006.

41 Kinoshita Satomi. Respecting the Wishes of Patients in Intensive Care Units. Nursing Ethics, 2007, 14(5):651-664.

42 Mc -Donagh; JR et al. Family satisfaction with family conferences about end-of-life care in the intensive care unit. Crit Care Med 2004; 32: 1584-8.

43 Atención de Enfermería Al Enfermo Terminal. Cuidados Paliativos. Ed. MAD. Colección Práctico profesional. Sevilla, 2008.

44 Protocolo de acuerdo Ministerio de Salud y Colegio de Enfermeras de Chile. Santiago, 2006.

45 Congreso Mundial de Medicina y Cuidados Intensivos. Declaración de Ética en Medicina Crítica y Cuidados Intensivos. Madrid, 1996

46 Ferrer Marta. Problemas más frecuentes de la enfermería en la terminalidad. En: Astudillo Wilson et al. Ob.cit.: 189-199.

\section{DIRECCIÓN PARA CORRESPONDENCIA}

Doctor: Francisco Javier León Correa

fleonc@uc.cl 